\title{
Practice of Menstrual Hygiene and Associated Factors among Adolescent School Girls in Dang District, Nepal
}

\author{
Chet Kant Bhusal iD \\ Department of Community Medicine, Universal College of Medical Science and Teaching Hospital, Tribhuvan University, \\ Bhairahawa, Rupandehi, Nepal \\ Correspondence should be addressed to Chet Kant Bhusal; bhusalck3112@gmail.com
}

Received 7 April 2020; Revised 22 June 2020; Accepted 6 July 2020; Published 24 July 2020

Academic Editor: Diego A. S. Silva

Copyright (C) 2020 Chet Kant Bhusal. This is an open access article distributed under the Creative Commons Attribution License, which permits unrestricted use, distribution, and reproduction in any medium, provided the original work is properly cited.

Background. Menstrual hygiene management has not been sufficiently addressed in developing countries. In many Nepalese societies, menstrual practices are still surrounded by sociocultural restrictions and taboos resulting in adverse health outcomes for adolescent girls. The purpose of this study was to determine menstrual hygiene practice and sociodemographic as well as socioeconomic factors associated with good menstrual hygiene practice amongst adolescent school girls in Dang district, Nepal. Methods. A cross-sectional study was conducted in Dang district, Nepal, among 406 adolescent girls studying in grades 8,9 , and 10 between ages of 10 and 19 years from April to October 2019. Randomly 5 units were selected from a total of 10 local units. After 5 units had been decided, 10 schools consisting of 5 government and 5 private schools were selected through a disproportionate stratified random sampling technique. A further 406 students were then selected randomly from the 10 selected schools. Bivariate analysis was used primarily to assess the association between dependent and independent variables and final measure of association was odds ratio. Variables which were associated with bivariate analysis were entered into a multivariable logistic regression model to identify associated factors of menstrual hygiene practice. Results. The mean age and family size were $15.13 \pm 1.19$ and $5.58 \pm 1.81$, respectively. A total of $272(67.0 \%)$ adolescents have good menstrual hygiene practice. Mothers and fathers with literature educational background (adjusted odds ratio $=0.52$, confidence interval: $0.30-0.89$ and $\mathrm{AOR}=2.55$, CI: $1.26-5.15$, respectively), family size greater than or equal to 5 ( $\mathrm{AOR}=0.61, \mathrm{CI}: 0.37-0.98)$, and living with relatives $(\mathrm{AOR}=0.45, \mathrm{CI}: 0.24-0.85)$ were significantly associated with good menstrual hygiene practice. Conclusions. Educational status of mother and father, family size, and living status were found to be independent associated factors of menstrual hygiene practice. In this context, this study demonstrates that administrators and policy makers should provide specific education regarding menstrual hygiene to both parents. Similarly local government needs to subsidize hygiene towels for school adolescents.

\section{Introduction}

Onset of menstruation is one of the vital changes happening in all females during their period of adolescence [1]. Menarche is not just a physiological process but it is a psychological, social, and behavioral transition from adolescence to womanhood [2]. Menstrual hygiene means necessities and requirements such as the use of sanitary pads or clean and soft absorbents, adequate washing of the genital area, proper disposal of used absorbents, and other special healthcare needs of women during monthly menstrual cycle $[3,4]$. In woman's life, good hygiene practice during menstruation is very important which prevents from adverse health outcomes $[5,6]$.

Though menstruation is a normal physiological process, it is still surrounded with social taboos, supernatural beliefs, misconceptions, and malpractices, which is very challenging for girls in developing countries $[7,8]$. Due to these social stigmatic, cultural, and religious restrictions, menstrual practices are regarded as big limitation for menstrual hygiene management [9]. Menstrual hygiene is very important; however it is still a neglected area of concern in many parts of the world [2]. Despite the fact that menstruation is a normal physiological process in females, it is not easy for 
every adolescent girl to maintain good hygiene [10]. Due to the lack of prior information about menstruation, girls experience different feelings such as fear, embarrassment, and guiltiness during their vaginal bleeding [11]. Adolescent girls particularly in developing countries still lack information about good menstrual hygiene practices [6]. Management of menstrual hygiene is considered as taboos in many parts of Nepal. Traditional and supernatural beliefs regarding menstruation may have negative impact on selfrespect, health, and education of adolescent girls [12]. Several studies have reported that infections may occur due to lack of hygiene during menstruation $[1,13]$. If menstrual period is not properly handled and safe hygiene is not practiced, this may lead to poor quality of life resulting from distress, reproductive tract infection, genitourinary tract infections, smelling, guiltiness, cervical cancer, poor academic performance, and school dropout [7, 14, 15]. Poor menstrual practice is also connected with many other complications such as premature births, stillbirths, miscarriages, infertility problems, and carcinoma of cervix [11]. Taboos and misconceptions on the subject of menstruating girls and menstrual hygiene develop in gender inequality and degradation of women empowerment [16]. Menstruation is regarded as a taboo even by the teachers; therefore, they do not offer information and guidance on the importance and management of menses. In developing countries, adolescent girls face various menstrual hygiene management challenges, especially at school [17].

Although there has been a growing literature on various aspects of menstrual hygiene, limited available studies in Nepal had focus on finding association between good menstrual hygiene practice and social factors among adolescent school girls. This is a hidden and unaddressed public health issue in country settings of Nepal. Therefore, this study was mainly conducted to identify menstrual hygiene practice and sociodemographic as well as socioeconomic factors associated with good menstrual hygiene practice amongst adolescent school girls in Dang district, Nepal. The result of this study might provide valuable information for health service providers, administrators, and policy makers in developing appropriate intervention programs. Moreover, the findings of this study will be used as baseline for further researcher.

\section{Materials and Methods}

2.1. Study Design and Source of Population. A cross-sectional study was conducted in Dang district, Nepal, from April to October 2019. School-going adolescent girls both from government and private schools studying in grades 8,9 , and 10 of age group 10-19 were included in the study. Similarly, girls whose menarche has not started, who had severe mental problems, and who were absent during the day of data collection were set as exclusion criteria.

2.2. Sample Size Determination and Sampling Technique. Sample size was calculated using formula $N=Z^{2} p q / L^{2}$ [18] with $95 \%$ level of confidence interval, and $6 \%$ margin of error; and $39.9 \%$ of respondents had practiced good menstrual hygiene [19]. Initial sample size of 256 was multiplied by design effect of 1.5 as multistage stratified probability random sampling was used. Thus sample size was 384 . Considering 5.5\% nonresponse rate, the final sample size was 406. A multistage probability random sampling was used as sampling technique among a total of 142 secondary schools of Dang district, Nepal. Among a total of 10 local units, 5 were selected randomly. Then, from selected 5 units, 10 schools consisting of 5 government and 5 private schools were selected by disproportionate stratified random sampling technique through nonreplacement lottery method. Further, 406 students were selected randomly from 10 schools which consisted of 41 from each government and 40 from each private school. Since sample size was 406, one additional student was taken from private school.

2.3. Data Collection Procedures and Validity. Data were collected using semistructured questionnaire by applying self-administered interview technique. Questionnaire was translated into Nepali and again retranslated into English language to find misinterpretation. The questionnaire was pretested among $10 \%$ of total sample residing in Bhairahawa, Rupandehi. Both English and Nepali version questionnaires were made and used according to familiarity of students. Two-day training was provided to three data collectors having qualification of Master's in Information Communication Technology, Bachelor in Public Health, and Bachelor of Science in Agriculture. All the filled questionnaires were reviewed and checked by the principal investigator on regular basis.

2.4. Data Processing and Analysis. Data were entered into Microsoft Excel and exported to Statistical Package for Social Science (SPSS) software version 20 for analysis. Characteristics of the sample were described using mean and standard deviation. Bivariate analysis was used primarily to assess the association between dependent and independent variables and those variables found to be associated with bivariate analysis $(p<0.05)$ were entered into the multivariate logistic regression model (stepwise backward likelihood ratio method) to identify the associated factors of practice of menstrual hygiene.

2.5. Setting. Dang district is located in inner Terai and mid hills of Rapti zone in the mid-western development region of Nepal. There are 2 submetropolitan cities, 1 municipality, and 7 rural municipalities in the district. The district has total population of 552,583 and annual population growth rate of 1.78 [20]. There are altogether 142 secondary schools including 86 private and 56 government; similarly there are total 24,622 students in secondary level in Dang district which includes 12,905 female and 11,717 male students [21].

2.6. Measurement of Practice on Menstrual Hygiene. The students' menstrual hygiene practice was assessed using 10 practice specific questions. Each correct response scored one 
TABLE 1: Distribution of background related characteristics of study population.

\begin{tabular}{|c|c|c|}
\hline General characteristics & Frequency $(n=406)$ & Percentage \\
\hline \multicolumn{3}{|l|}{ Age } \\
\hline $10-13$ years & 34 & 8.4 \\
\hline$>13$ Years & 372 & 91.6 \\
\hline \multicolumn{3}{|l|}{ Mean age $\pm S D ; 15.13 \pm 1.19$} \\
\hline \multicolumn{3}{|l|}{ Residence } \\
\hline Cities (submetropolitan city and municipalities) & 226 & 55.7 \\
\hline Village (rural municipalities) & 180 & 44.3 \\
\hline \multicolumn{3}{|l|}{ Religion } \\
\hline Hindu & 372 & 91.6 \\
\hline Non-Hindu (Christian and Muslim) & 34 & 8.4 \\
\hline \multicolumn{3}{|l|}{ Family type } \\
\hline Nuclear & 272 & 67.0 \\
\hline Joint and extended & 134 & 33.0 \\
\hline \multicolumn{3}{|l|}{ Family size } \\
\hline 1 to 4 & 120 & 29.6 \\
\hline 5 and above & 286 & 70.4 \\
\hline \multicolumn{3}{|l|}{ Type of school } \\
\hline Government & 205 & 50.5 \\
\hline Private & 201 & 49.5 \\
\hline \multicolumn{3}{|l|}{ Education of mother } \\
\hline Illiterate & 96 & 23.6 \\
\hline Literate & 310 & 76.4 \\
\hline \multicolumn{3}{|l|}{ Education of father } \\
\hline Illiterate & 37 & 9.1 \\
\hline Literate & 369 & 90.9 \\
\hline \multicolumn{3}{|l|}{ Occupation of mother } \\
\hline Homemaker & 193 & 47.5 \\
\hline Other than homemaker & 213 & 52.5 \\
\hline \multicolumn{3}{|l|}{ Occupation of father } \\
\hline Agriculture & 88 & 21.7 \\
\hline Other than agriculture & 318 & 78.3 \\
\hline
\end{tabular}

point, whereas wrong or do not know answer did not get score, and accordingly sum score was calculated out of 10 points as similarly described in previous studies [7]. The mean score was 7.07; therefore participants with a total score less than $7(0-6)$ were considered as having poor practice while those who scored 7-10 points were considered as having good practices.

2.7. Ethics Approval and Consent to Participate. Ethical approval was obtained from of Universal College of Medical Science and Teaching Hospital Institutional Review Committee (UCMS/IRC/063/19). Concerned stakeholders were officially contacted with letters and permission was obtained at all levels. The study was explained to participants. Since most of the respondents were below age group 18 years, verbal consent was obtained from children's parents/ guardians through telephone and after coordinating with parents, written informed consent was taken from school teachers before interview.

\section{Results}

The mean age and family size were $15.13 \pm 1.19$ and $5.58 \pm 1.81$, respectively. Out of total 406 school adolescents,

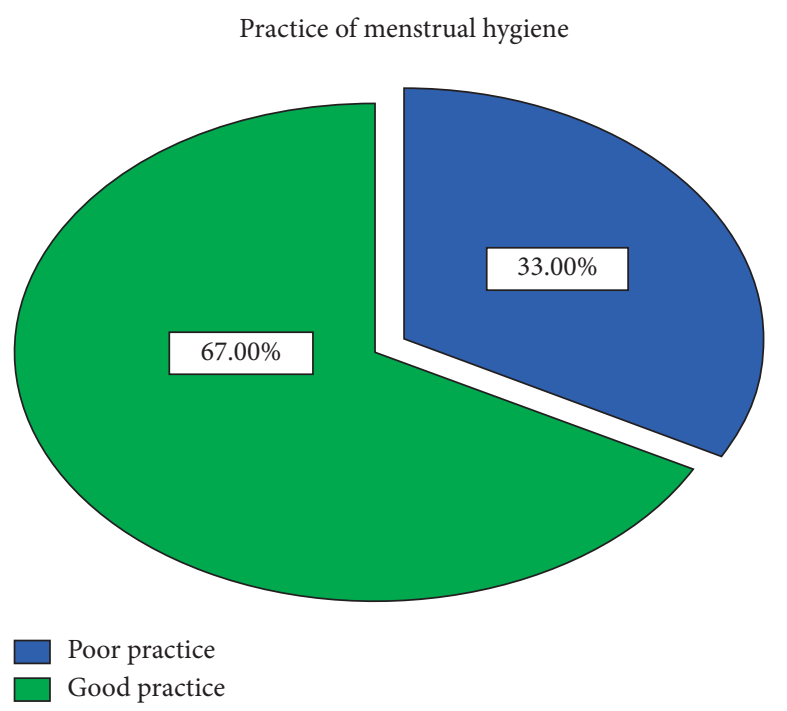

FIGURE 1: Distribution of adolescent school girls' practice grading on menstruation hygiene.

more than half $(55.7 \%)$ live in submetropolitan city and municipalities. Majority (91.6\%) of the adolescents followed Hindu religion. Two-thirds (67.0\%) of respondents had 
TABLE 2: Distribution of practice of adolescent school girls about menstruation hygiene and its management.

\begin{tabular}{lcc}
\hline Practice about menstruation and its management & Number $(n=406)$ & Percentage \\
\hline Used some form of absorbents during menstruation & 363 & 293 \\
Used sanitary pads during menstruation & 225 & 79.4 \\
Cleaning of genital organ with clean water & 67 & 55.4 \\
Daily bath during menstruation & 260 & 16.5 \\
Cleaning of genital organ at least three times a day & 311 & 64.0 \\
Changing of absorbent materials at least three times a day & 223 & 79.4 \\
Changing of absorbent materials in school & 340 \\
Drying of washed reusable clothes in direct sunlight & 343 \\
Disposed used pads in dustbins or toilets & 54.9 \\
Bathing with soap and water during menstruation & 83.7 \\
\hline
\end{tabular}

TABLE 3: Factors associated with level of practice in using bivariate and multivariate analysis.

\begin{tabular}{|c|c|c|c|c|c|}
\hline \multirow{2}{*}{ Characteristics } & \multicolumn{2}{|c|}{ Practice level (\%) } & \multirow{2}{*}{$p$ value } & \multirow{2}{*}{${ }^{\mathrm{a}} \mathrm{COR} 95 \% \mathrm{CI}$} & \multirow{2}{*}{${ }^{\mathrm{b}} \mathrm{AOR} 95 \% \mathrm{CI}$} \\
\hline & Poor practice & Good practice & & & \\
\hline \multicolumn{6}{|l|}{ Mother's education } \\
\hline Illiterate & $23(24.0)$ & $73(76.0)$ & $0.031^{*}$ & 1 & 1 \\
\hline Literate & $111(35.8)$ & $199(64.2)$ & & $0.57(0.33-0.95)$ & $0.52(0.30-0.89)$ \\
\hline \multicolumn{6}{|l|}{ Father's education } \\
\hline Illiterate & $20(54.1)$ & $17(45.9)$ & $0.004^{*}$ & 1 & 1 \\
\hline Literate & $114(30.9)$ & $225(69.1)$ & & $2.63(1.33-5.21)$ & $2.55(1.26-5.15)$ \\
\hline \multicolumn{6}{|l|}{ Father's occupation } \\
\hline Agriculture & $27(30.7)$ & $61(69.3)$ & 0.601 & 1 & - \\
\hline Other than agriculture & $107(33.6)$ & $211(66.4)$ & & $0.87(0.53-1.45)$ & \\
\hline \multicolumn{6}{|l|}{ Size of family } \\
\hline 1 to 4 members & $31(25.8)$ & $89(74.2)$ & $0.047^{*}$ & 1 & 1 \\
\hline 5 and above & $103(36.0)$ & $183(64.0)$ & & $0.62(0.38-0.99)$ & $0.61(0.37-0.98)$ \\
\hline \multicolumn{6}{|l|}{ Live with } \\
\hline Parents & $111(30.8)$ & $249(69.2)$ & & 1 & 1 \\
\hline Relatives & $23(50.0 \%)$ & $23(50.0 \%)$ & $0.009^{*}$ & $0.89(0.57-1.37)$ & $0.45(0.24-0.85)$ \\
\hline
\end{tabular}

*Significant at $p<0.05,1=$ reference category, ${ }^{a}$ crude odds ratio, and ${ }^{\mathrm{b}}$ adjusted odds ratio.

nuclear family. Nearly one-fourth $(23.6 \%)$ of the respondents' mothers were unable to read and write. Slightly less than half $(47.5 \%)$ of the mothers were homemakers. More than three-fourths $(78.3 \%)$ of respondents' fathers were engaged in occupations other than agriculture for their livelihood (Table 1).

Most of the girls, $363(89.4 \%)$, had used some form of absorbents during their menstruation. Nearly half (45.1\%) of the respondents did not dry washed reusable clothes in direct sunlight, most, $83.7 \%$, of the girls disposed used pads in dustbins or pit latrine. Only $16.5 \%$ of the school girls took daily bath during menstruation (Table 2).

The mean score of school girls' practice of menstruation and its hygienic management was $7.07 \pm 1.84$; therefore participants with a total score less than 7 (0-6) were considered as having poor practice while those who scored 7-10 points were considered as having good practices. Concerning the practice level of school-going adolescent girls, among 406 girls, two-thirds $(272(67.0 \%))$ practiced good menstrual hygiene whereas one-third (134 (33.0\%)) practiced poor menstrual hygiene (Figure 1).

Table 3 showed socioeconomic and sociodemographic factors associated with practice of menstrual hygiene. Those variables, which were found statistically significant with $p$ value lesser than or equal to 0.05 in bivariate analysis, were entered into the multivariate regression analysis model which identified mother's education, father's education, and size of family as associated factors with menstrual hygiene practice. Adolescent girls whose mothers were literate were 0.52 times less likely $(\mathrm{AOR}=0.52, \mathrm{CI}=0.30-0.88)$ to have good practices regarding menstrual hygiene and its management. However, the odds of having good menstrual hygiene practice were high among girls whose fathers were from literate backgrounds $(\mathrm{AOR}=2.61, \mathrm{CI}=1.31-5.23)$ and less among those who have 5 or more members in the family $(\mathrm{AOR}=0.61, \mathrm{CI}=0.37-0.98)$ (Table 3$)$.

\section{Discussion}

Literate mothers, literate fathers, size of family, and living with parents were associated factors of good menstrual hygiene practice in this study. The group of girls having 1 to 4 family members have good menstrual hygiene practice; this might be due to more interaction and communication with mothers in small families. More than three-fourths of school girls in developing countries like Nepal change absorbent materials in their school too. Less than one-fifth of the school girls took daily bath during menstruation. In this 
study, about two-thirds of school-going adolescent girls practiced good menstrual hygiene which is in line with studies done in Nepal [22] and Ethiopia [23]. However, several studies done in Ethiopia [24], Nigeria [25], and Ghana [26] found that higher proportion of the girls practiced good menstrual hygiene as compared to this study. In contrast to this study, small portion of good menstrual hygiene practice was also observed in several studies such as studies done in Nepal [12], Ethiopia [6, 19], and Nigeria [27]. This disparity might be due to different study setting, change in time, and divergence scoring system for measuring the practice level of menstrual hygiene in different studies.

Majority of adolescent girls in the study area used some form of the absorbents during their menstruation which is in line with previous study conducted in Ethiopia where $90.98 \%$ of school girls used some forms (sanitary pad and homemade clothes) during their menstrual period [8]. More than two-thirds of girls used disposable sanitary pads during their menstruation which is in concordance with the previous studies done in Nepal [28], India [29, 30], and Egypt [31]. However, several previous studies done in rural area of Nepal [32] and India [3, 33, 34] found that small portion of adolescent girls used sanitary pads during their menstruation. This might be due to the advancement in time and different study setting. This study revealed that more than half of the girls cleaned their genital organ with clean water during their menstruation; this is supported by the study done in Nepal [12] and India [35]. Less than onefifth of adolescent girls in the current study took bath daily during menstruation which is supported by the study done in Nepal [36] where only $14.3 \%$ of girls took bath more than 3 times in one menstrual period. Nearly two-thirds of the adolescent girls in this study cleaned their genital organ at least thrice a day; however, a study done in Amhara regional state, Ethiopia, found that smaller portion of the girls washed their genitalia at least three times per day [24]. On the top of that, another study done in Nagpur district, India, found that only one-third of girls cleaned their external genitalia two times or less a day [33]. The present study revealed that three-fifths of school girls practiced changing of absorbent materials at least three times a day which is in accordance with the study done in Uganda [37]. However, several other studies reported dissimilar result; one study done in rural area of Nepal found that around two-fifths of similar school girls changed absorbents at least three times per day [32] and another study done in Sokoto, Nigeria, found that higher portion $(70 \%)$ of the girls changed absorbents at least three times per day [38]. This might be due to time and focusing of rural area by previous study done in Nepal. In this study, more than three-fourths of respondent girls used to change absorbent materials in school; however, it is inconsistent with previous study conducted in Nagpur district of India where lower percentage of girls changed pads at school [33]. More than half of the adolescent girls in this study are drying washed reusable clothes in direct sunlight which is in line with the previous studies conducted in India [33] and Nigeria [39]. The present study reported that majority of the respondents disposed used menstrual materials in dustbins or pit latrine toilets which is accordance with the study done in Kathmandu valley of Nepal [40]. The majority of the girls in this study used to take bath with soap and water during their menstrual period which is in concordance with the study done in Northeast Ethiopia [8].

In the current study, mother's education was significantly associated with practice regarding menstrual hygiene which is also supported by several studies such as study done in India [39, 41], Adama Town, Ethiopia [6], Western Ethiopia [19], and Nigeria [38]. The study found significant association of practice of menstrual hygiene with father's education. Similar result was found in the bivariate analysis of another study done in rural area of West Bengal, India [41]. However, in contrast to this study, father's education was not found significant with practice of menstrual hygiene in another study done in India [42]. Positive association was found between good menstrual hygiene and size of family. The girls having 1 to 4 family members have good menstrual hygiene practice. There was positive association between good hygienic practice and living with parents which is in line with another study done in Nigeria [27]. This might reflect that girls who live with their parents discussed openly; they were better observed and guided during their menstrual period compared to those who reside with relatives.

\section{Limitations of the Study}

Studies from different parts of Nepal observed mean age of menarche as 13 years. As this study was conducted among adolescent girls studying in grades 8 to 10 , this might miss some young girls who are studying in lesser class and have just started their menarche. Therefore, further studies are recommended without excluding those younger girls.

\section{Conclusions}

One-third of the school-going adolescent girls practiced good menstrual hygiene. The main contributors of this are mother's education, father's education, family size, and living status of children. In this context, administrators and policy makers were recommended to provide specific education on menstrual hygiene to both parents. Similarly, local government needs to subsidize hygiene towels for school adolescents.

\section{Abbreviations \\ AOR: Adjusted odds ratio \\ CI: Confidence interval \\ OR: Odds ratio \\ SD: Standard deviation \\ SLC: School leaving certificate \\ SPSS: Statistical package for the social sciences.}

\section{Data Availability}

The raw data under identification policy will be provided upon request through e-mail to corresponding author. 


\section{Conflicts of Interest}

There are no conflicts of interest.

\section{Acknowledgments}

The author would like to express deepest gratitude to $\mathrm{Mr}$. Bhishma Bhusal, Ms. Bimala Kandel, Ms. Jenikshiya Lama, and Ms. Kavita Khanal, Bachelor in Public Health students, for their support in data collection. The author would like to acknowledge Bachelor in Public Health students, Mr. Rajan Gupta, Bivechana Chaulagain, Goma Sharma, Ranjita Dhungel, Alisha Shrestha, Bina Agrahari, and Surakhsya Poudel, for their assistance during data entry. Last but not least, the author would like to remember all participants of the study for their valuable time and support for providing inevitable information for the study.

\section{References}

[1] A. B. Mudey, N. Kesharwani, G. A. Mudey, and R. C. Goyal, "A cross-sectional study on awareness regarding safe and hygienic practices amongst school going adolescent girls in rural area of Wardha District, India," Global Journal of Health Science, vol. 2, no. 2, p. 225, 2010.

[2] D. J. Prajapati, J. P. Shah, and G. Kedia, "Menstrual hygiene: knowledge and practice among adolescent girls of rural Kheda district," Indian Journal of Community Medicine, vol. 13, no. 10 , p. 50, 2015

[3] T. N. Deshpande, S. S. Patil, S. B. Gharai, S. Patil, and P. Durgawale, "Menstrual hygiene among adolescent girls-A study from urban slum area," Journal of Family Medicine and Primary Care, vol. 7, no. 6, p. 1439, 2018.

[4] A. Singh, "Place of menstruation in the reproductive lives of women of rural North India," Indian Journal of Community Medicine, vol. 31, no. 1, p. 10, 2006.

[5] T. Bachloo, R. Kumar, A. Goyal et al., "A study on perception and practice of menstruation among school going adolescent girls in district Ambala Haryana, India," International Journal of Community Medicine And Public Health, vol. 3, no. 4, pp. 931-937, 2017.

[6] H. Anchebi, B. Shiferaw, R. Fite, and S. Abeya, "Practice of menstrual hygiene and associated factors among female high school students in adama town," Journal of Womens Health Care, vol. 6, no. 370, 2017.

[7] Z. Belayneh and B. Mekuriaw, "Knowledge and menstrual hygiene practice among adolescent school girls in southern Ethiopia: a cross-sectional study," BMC Public Health, vol. 19, no. 1, p. 1595, 2019.

[8] T. K. Tegegne and M. M. Sisay, "Menstrual hygiene management and school absenteeism among female adolescent students in Northeast Ethiopia," BMC Public Health, vol. 14, no. 1, p. 1118, 2014.

[9] R. Kaur, K. Kaur, and R. Kaur, "Menstrual hygiene, management, and waste disposal: practices and challenges faced by girls/women of developing countries," Journal of Environmental and Public Health, vol. 2018, 2018.

[10] T. Mahon and M. Fernandes, "Menstrual hygiene in South Asia: a neglected issue for WASH (water, sanitation and hygiene) programmes," Gender \& Development, vol. 18, no. 1, pp. 99-113, 2010.

[11] G. V. Bathija, D. D. Bant, and S. R. Itagimath, "Study on usage of woman hygiene kit among menstruating age group in field practice area of kims, hubli," International Journal of Biomedical Research, vol. 4, no. 2, pp. 94-98, 2013.

[12] R. N. Yadav, S. Joshi, R. Poudel, and P. Pandeya, "Knowledge, attitude, and practice on menstrual hygiene management among school adolescents," Journal of Nepal Health Research Council, vol. 15, no. 3, pp. 212-216, 2017.

[13] J. C. Bhatia and J. Cleland, "Self-reported symptoms of gynecological morbidity and their treatment in south India," Studies in Family Planning, vol. 26, no. 4, pp. 203-216, 1995.

[14] A. Dasgupta and M. Sarkar, "Menstrual hygiene: how hygienic is the adolescent girl?" Indian Journal of Community Medicine, vol. 33, no. 2, p. 77, 2008.

[15] A. Khanna, R. S. Goyal, and R. Bhawsar, "Menstrual practices and reproductive problems," Journal of Health Management, vol. 7, no. 1, pp. 91-107, 2005.

[16] A. Acharya, K. Yadav, and N. Baridalyne, "Reproductive tract infections/sexually transmitted infections in rural Haryana: experiences from the Family Health Awareness Campaign," Indian Journal of Community Medicine, vol. 31, no. 4, pp. 274-276, 2006.

[17] E. Korir, F. N. Okwara, and G. Okumbe, "Menstrual hygiene management practices among primary school girls from a pastoralist community in Kenya: a cross sectional survey," Pan African Medical Journal, vol. 31, no. 222, 2018.

[18] W. W. Daniel and C. L. Cross, Biostatistics: A Foundation for Analysis in the Health Sciences, Wiley, Hoboken, NJ, USA, 2018.

[19] S. P. Upashe, T. Tekelab, and J. Mekonnen, “Assessment of knowledge and practice of menstrual hygiene among high school girls in Western Ethiopia," BMC Women's Health, vol. 15, no. 1, p. 84, 2015.

[20] Nepal Go, "Government of Nepal national planning commission secretariat central bureau of statistics," 2012, http:// www.cbs.gov.np.

[21] DEO. District education office Dang annual report. http:// www.deodang.gov.np/allcontent/Detail/114.

[22] M. S. Neupane, K. Sharma, A. P. Bista, S. Subedi, and S. Lamichhane, "Knowledge on menstruation and menstrual hygiene practices among adolescent girls of selected schools, Chitwan," Journal of Chitwan Medical College, vol. 10, no. 1, pp. 69-73, 2020.

[23] B. Kitesa, T. Getahun, and K. Wako, "Assessment of knowledge and practice of adolescent in-school girls towards menstrual hygiene management and determining factors in lucy village of Ethiopian great rift valley, 2016," International Journal of Immunology, vol. 4, no. 6, pp. 52-63, 2016.

[24] T. K. Gultie, "Practice of menstrual hygiene and associated factors among female Mehalmeda high school students in Amhara Regional State, Ethiopia," Science Journal of Public Health, vol. 2, no. 3, pp. 189-195, 2014.

[25] U. M. Lawan, M. Yusuf, and A. B. Musa, "Menstruation and menstrual hygiene amongst adolescent school girls in Kano, Northwestern Nigeria," African Journal of Reproductive Health, vol. 14, no. 3, pp. 201-207, 2010.

[26] E. P. K. Ameade and H. A. Garti, "Relationship between female university students' knowledge on menstruation and their menstrual hygiene practices: a study in Tamale, Ghana," Advances in Preventive Medicine, vol. 2016, Article ID 1056235, 10 pages, 2016.

[27] F. O. Fehintola, A. O. Fehintola, A. O. Aremu, A. Idowu, O. A. Ogunlaja, and I. P. Ogunlaja, "Assessment of knowledge, attitude and practice about menstruation and menstrual hygiene among secondary high school girls in Ogbomoso, Oyo state, Nigeria," International Journal of Reproduction, 
Contraception, Obstetrics and Gynecology, vol. 6, no. 5, pp. 1726-1732, 2017.

[28] S. Karki, A. K. Rajbhandari, M. Dahal, P. Shahi, and S. Sharma, "Knowledge and practice on menstrual hygiene among adolescent girls of selected slums in Kathmandu valley," Journal of Patan Academy of Health Sciences, vol. 5, no. 2, pp. 114-122, 2018.

[29] K. Ramachandra, S. Gilyaru, A. Eregowda, and S. Yathiraja, "A study on knowledge and practices regarding menstrual hygiene among urban adolescent girls," International Journal of Contemporary Pediatrics, vol. 3, no. 1, pp. 142-145, 2016.

[30] S. Omidvar and K. Begum, "Factors influencing hygienic practices during menses among girls from south India-A cross sectional study," International Journal of Collaborative Research on Internal Medicine \& Public Health, vol. 2, no. 12, 2010.

[31] A.-H. El-Gilany, K. Badawi, and S. El-Fedawy, "Menstrual hygiene among adolescent schoolgirls in Mansoura, Egypt," Reproductive Health Matters, vol. 13, no. 26, pp. 147-152, 2005.

[32] D. Sapkota, D. Sharma, H. Pokharel, S. S. Budhathoki, and V. K. Khanal, "Knowledge and practices regarding menstruation among school going adolescents of rural Nepal," Journal of Kathmandu Medical College, vol. 2, no. 3, pp. 122-128, 2013.

[33] S. B. Thakre, S. S. Thakre, M. Reddy, N. Rathi, K. Pathak, and S. Ughade, "Menstrual hygiene: knowledge and practice among adolescent school girls of Saoner, Nagpur district," Journal of Clinical and Diagnostic Research, vol. 5, no. 5, pp. 1027-1033, 2011.

[34] R. Juyal, S. Kandpal, J. Semwal, and K. Negi, "Practices of menstrual hygiene among adolescent girls in a district of Uttarakhand," Indian Journal of Community Health, vol. 24, no. 2, pp. 124-128, 2012.

[35] B. Arumugam, S. Nagalingam, P. M. Varman, P. Ravi, and R. Ganesan, "Menstrual hygiene practices: is it practically impractical?" International Journal of Medicine and Public Health, vol. 4, no. 4, 2014.

[36] P. Amatya, S. Ghimire, K. E. Callahan, B. K. Baral, and K. C. Poudel, "Practice and lived experience of menstrual exiles (Chhaupadi) among adolescent girls in far-western Nepal," PloS One, vol. 13, no. 12, 2018.

[37] G. Miiro, R. Rutakumwa, J. Nakiyingi-Miiro et al., "Menstrual health and school absenteeism among adolescent girls in Uganda (MENISCUS): a feasibility study," BMC Women's Health, vol. 18, no. 1, p. 4, 2018.

[38] M. Oche, A. Umar, G. Gana, and J. Ango, "Menstrual health: the unmet needs of adolescent girls' in Sokoto, Nigeria," Scientific Research and Essays, vol. 7, no. 3, pp. 410-418, 2012.

[39] R. Sudeshna and D. Aparajita, "Determinants of menstrual hygiene among adolescent girls: a multivariate analysis," National Journal of Community Medicine, vol. 3, no. 2, pp. 294-301, 2012.

[40] B. Pokhrel, S. Mahat, and K. Parajuli, "Knowledge and practice regarding menstrual hygiene among physically disabled women in Kathmandu, Nepal," Journal of Karnali Academy of Health Sciences, vol. 3, no. 1, pp. 1-10, 2020.

[41] I. Sarkar, M. Dobe, A. Dasgupta, R. Basu, and B. Shahbabu, "Determinants of menstrual hygiene among school going adolescent girls in a rural area of West Bengal," Journal of Family Medicine and Primary Care, vol. 6, no. 3, p. 583, 2017.

[42] P. R. Kokiwar, B. Ramesh, P. S. Reddy, P. Vindhya, R. Nischala, and P. S. Komal, "Menstrual hygiene among school going adolescent girls: a comparative study among government and private schools," Indian Journal of Preventive Medicine, vol. 6, no. 1, 2018. 